\title{
Two-nucleon transfer between heavy ions
}

from P. E. Hodgson

THE theoretical analysis of reactions between heavy ions in which two nucleons are transferred from the projectile to the target has now been developed to a high degree of sophistication. The basic distorted wave theory was formulated many years ago and successfully applied to analyse (d,p) reactions. But in the case of heavy ion reactions many of the approximations that are made for $(d, p)$ reactions are no longer valid, in particular the use of a zero-range interaction and the neglect of recoil. Inclusion of these effects is very complicated and greatly increases the computation time. Furthermore it has become increasingly clear that in many transfer reactions involving collective nuclei the contributions from two-step processes that take place through the pre-excitation of the target nucleus or by post-excitation of the residual nucleus must also be included. Lastly in heavy ion reactions there are substantial contributions from a large range of incident orbital angular momenta.

All these factors have combined to make the whole calculation prohibitively long, even on the fastest computer,

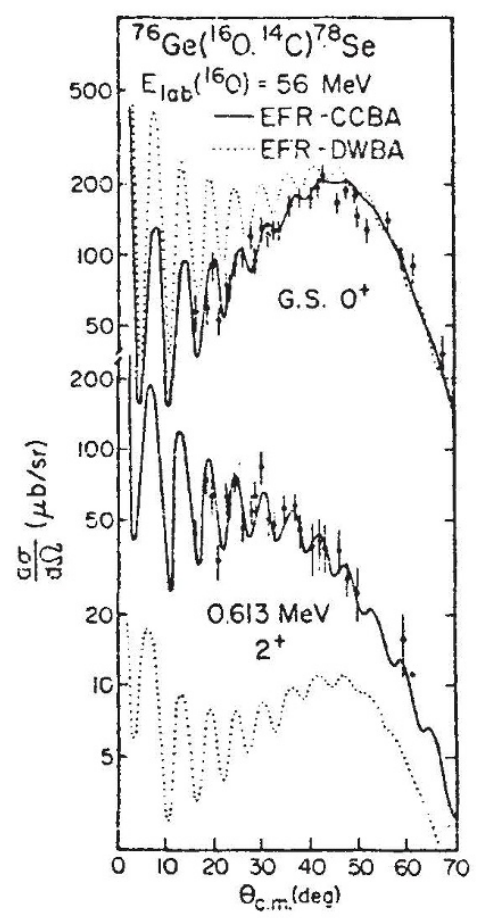

Differential cross section for the twoproton transfer reaction ${ }^{76} \mathrm{Ge}\left({ }^{16} \mathrm{O},{ }^{14} \mathrm{C}\right)$ ${ }^{78} \mathrm{Se}$ to the ground and $2^{+}$excited state of ${ }^{78} \mathrm{Se}$ compared with exact finite range calculations. The dotted line shows the one-step distorted wave calculation (EFR-DWBA) and the full line shows the coupled channels calculation (EFR-CCBA) that takes into account the contributions of two-step processes in the exit channel. if it is carried out in the usual way. Recently, however, Low and Tamura (Phys. Lett., 48B, 285; 1974) have developed new techniques for evaluating the multidimensional integrals that occur in these calculations, and these have so strikingly reduced the computing time that these calculations are now practicable.

These new computing techniques have been applied by Tamura, Low and Udagawa (Phys. Lett., 51B, 116; 1974) to calculate the cross section for the two nucleon transfer reaction ${ }^{76} \mathrm{Ge}\left({ }^{16} \mathrm{O}\right.$, $\left.{ }^{14} \mathrm{C}\right)^{78} \mathrm{Se}$ to the ground and first excited $\left(2^{+}\right)$state of the final nucleus. Comparison with the measurements of Lemaire, Mermaz, Sztark and Cunsolo shows that the distorted wave calculations are inadequate, even when finite range effects are taken into account (see dotted line in figure). The cross section for the reaction to the ground state shows too much oscillation and is too high at small angles, while that of the reaction to the $2^{+}$state at 0.613 $\mathrm{MeV}$ is far too small.

They then took into account the inelastic excitation in the exit channel. The residual nucleus ${ }^{78} \mathrm{Se}$ is easily excited into low-lying collective states, so it is possible for the two transferred nucleons to be first added to the target to give ${ }^{78} \mathrm{Se}$ in its ground state, and then for the outgoing ${ }^{14} \mathrm{C}$ nucleus to interact again and excite the ${ }^{78} \mathrm{Se}$ nucleus to the $2^{+}$state. This two-step contribution to the reaction adds to the direct contribution when the two nucleons are transferred so as to produce the ${ }^{78} \mathrm{Se}$ nucleus immediately in its $2^{+}$state. There is also a small effect on the cross section of the reaction to the ground state, for this similarly has a two-step component that goes first to the $2^{+}$state, which is then de-excited by inelastic scattering in the exit channel.

This complicated calculation can be carried out without any adjustment of parameters. The wave function of the two transferred protons to be added to the target was obtained using the standard Bardeen-Cooper-Schreiffer method with the Random Phase Approximation. The single-particle energies were taken from previous work and the strength of the pairing interaction was determined by fitting the experimental separation energy. The separation of the two protons from the ${ }^{16} \mathrm{O}$ was calculated using the fractional parentage coefficients of Cohen and Kurath.

The results of this calculation, taking the two-step processes in the exit channel into account, are shown by the full line in the figure. The agreement is excellent, indicating that the essential physics of the process is now well under- stood. In particular, it is clear why the inclusion of the two-step processes produces such a dramatic improvement. Since the ${ }^{78} \mathrm{Se}$ and ${ }^{76} \mathrm{Ge}$ nuclei are both superconducting, the transition from the ground state of the target to the ground state of the final nucleus is much stronger than the transition to the first excited state. Thus inclusion of the two-step process greatly enhances the $2^{+}$cross section but has a much smaller, though still important, effect on the reaction to the ground state.

There are still more effects that could be taken into account, in particular the inelastic effects in the entrance channel. In the calculation of the form factor of the transferred protons only the most important term, corresponding to $0 \mathrm{~s}$ relative motion of the two protons, was taken into account, and a fuller calculation could investigate the contribution of the higher order terms. Nevertheless, the success of the present calculations suggests that the most important effects have already been included, and that these improvements will not substantially affect the detailed understanding of this reaction that has now been achieved.

\section{Gels and chains}

from a Correspondent

IT has been known, since the work of the Singers and of Allison in the $1950 \mathrm{~s}$, that gel formation in solutions of deoxy sickle-cell haemoglobin $(\mathrm{Hb}-\mathrm{S})$ is highly dependent on $\mathrm{Hb}-\mathrm{S}$ concentration and that the minimum gelling concentration (MGC) is a function of temperature, $p \mathrm{H}$, ionic strength and the presence of organic phosphates which bind preferentially to deoxy $\beta$-chains. More recent studies by Bookchin, Nagel and Ranney on mixtures of deoxy $\mathrm{Hb}-\mathrm{S}$ with other haemoglobins showed that the MGC values were higher, but that the partial concentrations of $\mathrm{Hb}-\mathrm{S}$ at gelation were lower, indicating the incorporation of other haemoglobin species in the gel. Following an earlier analysis of these results by Minton on the basis of a two-step aggregation process, they have now been re-interpreted by Moffat (Science, 185, 274; 1974), assuming the formation of hybrid tetramers formed by association of unlike dimers in such binary mixtures, for example $\alpha^{\mathrm{A}}{ }_{2} \beta^{\mathrm{A}} \beta^{\mathrm{S}}$ from $\alpha^{\mathrm{A}} \beta^{\mathrm{A}}$ and $\alpha^{\mathrm{A}} \beta^{\mathrm{S}}$ in a mixture of $\mathrm{Hb}-\mathrm{S}$ and normal adult $\mathbf{H b}-\mathbf{A}$.

According to Moffat all possible tetramer species are in principle capable of incorporation into the gel: the gel forms when the weighted sum of their concentrations reaches a critical value, the MGC for pure deoxy $\mathrm{Hb}-\mathrm{S}$ under the same conditions, which is assigned 